\title{
Olfactory impairment in autoimmune encephalitis: another piece of the puzzle
}

\author{
Alessandra Morano ${ }^{1} \cdot$ Emanuele Cerulli Irelli ${ }^{1}$ Martina Fanella ${ }^{2}$. Biagio Orlando ${ }^{1} \cdot$ Enrico Michele Salamone $^{1}$. \\ Emanuele Tinelli ${ }^{3}$. Gabriele Ruffolo ${ }^{4,5} \cdot$ Luigi Zuliani $^{6}$. Jinane Fattouch ${ }^{1} \cdot$ Mario Manfredi $^{1}$. \\ Anna Teresa Giallonardo ${ }^{1}$. Carlo Di Bonaventura ${ }^{1}[0$
}

Received: 8 October 2021 / Revised: 31 December 2021 / Accepted: 31 December 2021 / Published online: 10 January 2022

(c) The Author(s), under exclusive licence to Springer-Verlag GmbH Germany 2022

\begin{abstract}
Background Despite being long neglected, olfaction has recently become a focus of intense research in neuroscience, as smell impairment has been consistently documented in both neurodegenerative and neuroinflammatory diseases. Considering the close anatomo-functional correlations between the limbic system and the central olfactory structures, we investigated olfaction in a population of patients with autoimmune encephalitis (AE).

Methods Nineteen adult subjects (14 males, median age 64 years) diagnosed with definite (14/19) or possible (5/19) AE and followed for $\geq 6$ months were enrolled. The Brief Smell Identification Test (B-SIT), a 12-item, forced-choice, scratchand-sniff measure, was used to assess the patients' olfactory function in comparison with a group of sex- and age-matched healthy controls $(\mathrm{HC})$. According to the B-SIT score, subjects were classified as anosmic $(<6)$, hyposmic $(6-8)$ and normal $(\geq 9)$. Electro-clinical, laboratory and neuroimaging findings were reviewed.

Results Smell impairment was revealed in 15/19 patients (9 hyposmic, 6 anosmic), compared with 5/19 HC ( $p=0.0029$ ). Age, gender and smoking habits did not affect the participants' performance at B-SIT. Olfactory dysfunction appeared more common among patients with definite AE $(p=0.0374)$, regardless of autoantibody status. Subjects with higher modified Rankin Scale (mRS) scores at AE onset more likely presented hyposmia/anosmia $(p=0.033)$, and so did those with bilateral ictal/interictal EEG abnormalities $(p=0.006)$.

Conclusions We found olfaction to be impaired in a significantly large proportion of AE cases. Smell deficits appeared more common in subjects with severe $\mathrm{AE}$ (as indicated by both definite diagnosis and higher mRS score), and might represent an additional feature of immune-mediated encephalitis.
\end{abstract}

Keywords Smell impairment $\cdot$ Anosmia $\cdot$ Autoimmune encephalitis $\cdot$ Limbic system $\cdot$ Neuroinflammation

Carlo Di Bonaventura

c_dibonaventura@yahoo.it

1 Epilepsy Centre, Department of Human Neurosciences, "Sapienza" University of Rome, Viale dell’Università, 30, 00185 Rome, Italy

2 Neurology Unit, Ospedale “San Camillo de' Lellis", Rieti, Italy

3 Neuroradiology Unit, "Sapienza" University of Rome, Rome, Italy

4 Department of Physiology and Pharmacology, Istituto Pasteur-Fondazione Cenci Bolognetti, "Sapienza" University of Rome, Rome, Italy

5 IRCCS San Raffaele Pisana, Rome, Italy

6 Neurology Unit, AULSS8 Berica, Vicenza, Italy

\section{Introduction}

Olfaction is the most ancient of all senses and the most conserved along evolution, despite remarkable inter-species differences [1]. Notwithstanding, smell has been long neglected, based on the assumption that humans are "microsmatic", i.e. capable of limited odor discrimination compared with other mammals, and that olfaction has progressively lost its usefulness in activities crucial for survival such as food search and mating. In fact, humans have an extensive odor repertoire, and olfactory dysfunctions may significantly affect health and quality of life, as dramatically brought to the public attention by the recent SARS-CoV2 pandemic [2]. Moreover, smell loss has been recognized as an early manifestation of various neurodegenerative disorders [3], 
in particular Parkinson's and Alzheimer's disease, providing not only a useful clinical predictor but also possible insights into their pathophysiological mechanisms. Fewer studies have also described olfactory alterations related to neuroimmunological disorders, such as systemic lupus erythematosus, multiple sclerosis and neuromyelitis optica [4]. Finally, smell impairment has been documented through a psychophysical test in a single cohort of subjects diagnosed with autoimmune encephalitis (AE) [5], and hyposmia has been recently reported as a possible manifestation of the newly recognized anti-adenylate kinase 5 (AK5) encephalitis [6]. Following these findings, we assessed the olfactory function of $19 \mathrm{AE}$ patients, comparing them with a group of sex- and age-matched healthy controls (HC), to confirm olfactory deficits in this patient population and investigate possible correlations between olfaction and electro-clinical and neuroimaging findings.

\section{Materials and methods}

\section{Study participants}

The study participants were identified among adult subjects diagnosed with AE from 2011 to 2019 at the Neurology Department of Policlinico "Umberto I" of Rome. The diagnosis of possible/probable seronegative/definite $\mathrm{AE}$ was confirmed according to the current criteria by Graus et al. [7]. Only patients followed up at the neurology outpatient service for $\geq 6$ months after $\mathrm{AE}$ onset were enrolled.

\section{Study procedures}

The Brief Smell Identification Test (B-SIT) (Sensonics International, Haddon Heights, $\mathrm{NJ}$ ) was administered to all participants from June to early November 2019, on their scheduled follow-up visits. The B-SIT is a validated, crosscultural, 12-item, four alternative forced-choice measurement, consisting of a booklet where each page contains a strip embedded with a microencapsulated odorant. After thoroughly explaining the procedure, for each of the 12 items, the examiner scratched the pad with a pencil provided by the manufacturer to release the odorant, and then placed it under the subject's nose (birhinally) for five seconds. After that, the participant was presented with four alternatives (translated into Italian by the examiner) and was asked to choose the most likely one. Based on previous literature [8], the following categories were identified according to the B-SIT score: anosmia (B-SIT <6), hyposmia (B-SIT 6-8) and normal olfaction (B-SIT $\geq 9$ ). Before the procedure, the examiner interviewed the study subjects about smoking habits, symptoms suggestive for concomitant nasal conditions (e.g. infectious or allergic rhinitis) and olfactory complaints (e.g. diminished or amplified sense of smell, cacosmia). Subjective alterations of taste were also recorded although they were not further investigated. During the same session, all participants also completed the Montreal Cognitive Assessment (MoCA) test. The score of 26 was used as a cut-off to define cognitive impairment based on prior validated data [9]. Finally, within one month from B-SIT all patients underwent anterior rhinoscopy performed through a nasal speculum by an expert otorhinolaryngologist, to rule out major nasal conditions.

Nineteen sex- and age-matched HC (13 males, median age 65 years, range 32-74) were recruited among the hospital staff and their friends and relatives, and tested via B-SIT from June to July 2021. HC had neither prior SARSCoV2 infection nor current self-reported smell alterations (Supplementary Table 2).

The participants gave their informed consent to the study, which was approved by the Ethics Committee of Policlinico "Umberto I" of Rome.

\section{Data collection}

Demographics, clinical features, laboratory and EEG findings were collected through medical chart review and carefully analyzed. All patients had already been tested for onconeural (anti-Yo, Ri, Hu, Ma2, Amphiphysin, CV2/CRMP5) autoantibodies (Abs), Abs directed against neuronal surface antigens [N-methyl-D-aspartate receptor(NMDAR), leucine-rich glioma-inactivated 1 (LGI11), contactin-associated protein-like 2 (CASPR2), alpha-amino-3-hydroxyl-5-methyl-4-isoxazole-propionate receptor (AMPAR), $\gamma$-aminobutyric acid receptor $(\mathrm{GABAR})_{\mathrm{B}}$, dipeptidyl-peptidase-like protein 6 (DPPX)] and anti-glutamic acid decarboxylase (GAD) $65 \mathrm{Abs}$, whereas anti-GABA $A_{A} R$ and anti-SRY-Box Transcription Factor 1 (SOX1) Abs were tested in one case each. Brain MRI scans performed during the year preceding the administration of B-SIT were revised by an expert neuroradiologist to evaluate the presence and the lateralization of typical $\mathrm{AE}$ findings, i.e. T2/FLAIR hyperintensity involving the mesial temporal structures, with or without concomitant volume changes of either hippocampus or amygdala.

\section{Statistical analysis}

Data were tested for normal distribution using the Shapiro-Wilk test, resulting in generalized non-normal distribution, and therefore were presented as median and interquartile range (IQR). The comparison of B-SIT scores between cases (AE patients) and controls (HC) was performed through Mann-Whitney $U$ test, whereas Fisher's exact test was used to compare the proportion of subjects with hyposmia/anosmia in each group. In AE patients, the comparison 
between relevant groups (smell impaired vs normal olfaction) was performed through Mann-Whitney $U$ test in case of continuous variables (e.g. age, diagnostic delay, modified Rankin scale score-mRS), whereas categorical data (e.g. sex, Abs status, smoking habits, cognitive impairment) were compared through Fisher's exact test. Group tests were two sided with $p<0.05$ considered statistically significant. IBM SPSS Statistics version 25 for Windows (IBM Corp, Armonk, NY, USA) was used for data analysis.

\section{Results}

\section{Patient demographics and general characteristics}

Nineteen patients (14 males) with a median age of 64 years (range 28-76, IQR 58-72 years) were included in the study. The diagnosis of definite AE was confirmed in 14/19 subjects, whereas five out of 19 were classified as having possible AE, according to the current criteria (for details, see Supplementary Table 1) [7]. All patients had limbic encephalitis except for one who was diagnosed with anti-NMDAR encephalitis based on clinical features and anti-NMDAR Abs detected in both CSF and serum. Specific autoantibodies were found in other 8 participants, namely anti-LGI1 in four, anti-CASPR2 in three, anti-SOX1 in one. The median diagnostic delay was 4.5 months (range 0.5-48 months) and the median follow-up at B-SIT was 37 months (range 6-96 months). All patients but one received immunotherapy (IT) at the time of diagnosis and 14/19 developed autoimmune-associated epilepsy (Table 1).

\section{Olfactory complaints and risk factors for smell loss}

Three patients complained of persistent smell loss when BSIT was administered (one of these subjects also had olfactory/gustatory seizures), whereas two recalled reduced sense of smell at the time when the other AE-related symptoms (mainly seizures and memory deficits) began; such symptom apparently resolved before the diagnosis was even made and IT was started. Conversely, two subjects reported dysosmias (in particular, unpleasantly amplified odors) at AE onset, which gradually subsided. In addition, another patient (already published) [10] not only complained of early cacosmia and ageusia that partly improved over time, but also presented drug-resistant focal seizures characterized by piloerection with/without concomitant olfactory hallucinations, either spontaneous or triggered by emotionally charged odors (e.g. his wife's perfume).

Six patients were smoking at the time of B-SIT, and other six had a history of smoking habit. Additional potential risk factors for smell impairment were revealed in four cases (i.e. obstructive sleep apnea in three, septoplasty in
Table 1 General characteristics of the patient population ( $n$ 19)

\begin{tabular}{|c|c|}
\hline \multicolumn{2}{|l|}{ General characteristics } \\
\hline \multicolumn{2}{|l|}{ Age at AE onset (years) } \\
\hline Median, [range] & $59[21-75]$ \\
\hline \multicolumn{2}{|l|}{ Gender } \\
\hline Female (\%) & $5(26.3)$ \\
\hline Male $(\%)$ & $14(73.7)$ \\
\hline \multicolumn{2}{|l|}{ Relevant data in medical history } \\
\hline Neoplasms & $6^{\S}$ \\
\hline Previous & 3 \\
\hline Concomitant & 3 \\
\hline ENT comorbidities & 4 \\
\hline \multicolumn{2}{|l|}{ Clinical presentation at $\mathrm{AE}$ onset (\%) } \\
\hline Seizures & $19(100)$ \\
\hline Cognitive impairment & $13(68.4)$ \\
\hline Mood/behavioral disorders & $18(94.7)$ \\
\hline Sleep disorders & $9(47.4)$ \\
\hline \multicolumn{2}{|l|}{$\mathrm{APE}^{2}$ score } \\
\hline$<4$ & 1 \\
\hline $4-6$ & 11 \\
\hline$\geq 7$ & 7 \\
\hline \multicolumn{2}{|l|}{ Laboratory findings } \\
\hline \multicolumn{2}{|l|}{ CSF findings } \\
\hline Hyperprot/hypercell & 10 \\
\hline Normal & 6 \\
\hline Not available & 3 \\
\hline \multicolumn{2}{|l|}{ AutoAbs profile (CSF/serum) } \\
\hline Seropositive & 9 \\
\hline Anti-LGI1 & 4 \\
\hline Anti-CASPR2 & 3 \\
\hline Anti-NMDAR & 1 \\
\hline Anti-SOX1 & 1 \\
\hline Seronegative & 10 \\
\hline \multicolumn{2}{|l|}{ MRI findings ${ }^{\mathbb{I}}$} \\
\hline Unilateral $\mathrm{mT}$ & 7 \\
\hline Bilateral mT & 10 \\
\hline Multifocal & 1 \\
\hline Normal & 1 \\
\hline \multicolumn{2}{|l|}{ EEG data } \\
\hline Ictal EEG pattern & 9 \\
\hline Interictal EEG findings & 14 \\
\hline IEDs & 11 \\
\hline Slow activity & 3 \\
\hline Diagnostic delay (mo), median, [range] & $4.5[0.5-48]$ \\
\hline \multicolumn{2}{|l|}{ Response to immunotherapy } \\
\hline Yes/partial & 16 \\
\hline No/not applicable & 3 \\
\hline \multicolumn{2}{|l|}{ Sequelae at follow-up (\%) } \\
\hline Autoimmune epilepsy & $14(73.7)$ \\
\hline Cognitive impairment & $8(42.1)$ \\
\hline Mood disorders & $10(52.6)$ \\
\hline
\end{tabular}


Table 1 (continued)

\begin{tabular}{ll}
\hline General characteristics & \\
\hline Olfaction-related features & \\
Age at BSIT (years) & 64 [28-76] \\
Median age [range] & \\
Delay to BSIT (months) & $42[6-96]$ \\
Mean [range] & \\
Smoking habit at B-SIT & 6 \\
Current smokers & 6 \\
Previous smokers &
\end{tabular}

$A b s$ antibodies, $A E$ autoimmune encephalitis, $B$-SIT Brief Smell Identification Test, CASPR2 contactin-associated protein-like 2, CSF cerebrospinal fluid, $E N T$ ear, nose and throat, IED interictal epileptiform abnormalities, LGI-1 leucine-rich glioma-inactivated 1, $m T$ mesial temporal, n.a. not applicable, NMDAR N-methyl-D-aspartate receptor, SOX1 SRY-Box Transcription Factor 1

${ }^{\S}$ Of the 6 patients with history of neoplasms, only one ever received chemotherapy (hydroxyurea for essential thrombocythemia)

I $\mathrm{APE}^{2}$ score as defined in Dubey et al., J Neuroimmunol. 2018

II MRI alterations are defined as T2/FLAIR hyperintensity with or without volume changes of the mesial temporal (mT) structures

\section{B-SIT score in AE vs HC}



Fig. 1 The significant difference between patients' and controls' performance at B-SIT. The dotted line indicates the cut-off for normal olfactory function. $A E$ autoimmune encephalitis, B-SIT Brief Smell Identification Test, $H C$ healthy controls

one). No patient reported previous head trauma. None of the participants was experiencing nasal conditions on the day B-SIT was administered. Rhinoscopy was unremarkable in all cases.

\section{Olfactory measures and anatomo-electro-clinical correlations}

Fifteen out of 19 (78.9\%) patients (11 men) scored $<9$ at B-SIT - qualifying as olfactory impaired-compared with $5 / 19$ HC $(p=0.0029)$ (Fig. 1). As to the severity of the smell deficit, hyposmia was diagnosed in 9/15 subjects and anosmia in 6/15, whereas no HC was classified as anosmic. Indeed, the median BSIT score was significantly lower in AE patients compared with HC (AE: BSIT score $=7$, IQR 5-8 vs HC: $\mathrm{BSIT}$ score $=10$, IQR $8-11, p<0.001)$. Age, gender, smoking habit (when considering both current smokers and all-time smokers) and additional risk factors for smell loss did not appear to affect olfactory function. Smell impairment was significantly more common in patients diagnosed with definite $\mathrm{AE}$ than those with possible $\mathrm{AE}(p=0.0374)$. However, no difference was revealed according to Abs status (seropositive versus seronegative). Interestingly, subjects with higher $\mathrm{mRS}$ scores at AE onset were significantly more likely to present either hyposmia or anosmia $(p=0.033)$. The diagnostic delay appeared longer in normal patients (median seven months) compared with impaired ones (median four months), although it did not reach statistical significance. Among 16 participants undergoing cerebrospinal fluid (CSF) analysis, inflammatory changes were detected in seven, all presenting with smell deficit. Finally, no significant correlation was found between the lateralization of the MRI-detected mesial temporal alterations and the patients' performance at B-SIT, although bilateral involvement was more frequently observed among olfactory impaired subjects than normal ones (10/15 versus $1 / 4)$. However, when considering the lateralization of ictal/interictal EEG findings, a significant difference was documented $(p=0.006)$, with hyposmic/anosmic patients more often presenting bilateral EEG abnormalities (Table 2).

\section{Discussion}

Autoimmune encephalitis is a recently recognized entity, whose phenotypic spectrum is still to be fully elucidated. The core clinical manifestations observed in most AE cases definitely reflect the prominent involvement of the limbic system, and include cognitive (memory) deficits, behavioral/ psychiatric disorders and temporal lobe seizures. The present study suggests that olfaction impairment may represent another common (but underreported) feature of AE.

Indeed, it is widely acknowledged that odors can evoke recollections of the past more intensely than any other sensation, especially within an emotionally charged context [11]. The reason for such close relationship between smell, memory and emotion lies in the "depth" of the human brain, where the phylogenetically ancient structures subserving these functions (i.e. paleopallium and archipallium) are nestled, with their dense network of reciprocal connections. Based on these anatomo-functional premises, it appeared only likely that AE might affect olfaction as well as memory and mood. 
Table 2 Correlations between smell impairment and clinical/ instrumental data

\begin{tabular}{|c|c|c|c|}
\hline Patients' characteristics & BSIT < 9 (15) & $\mathrm{BSIT} \geq 9$ (4) & Significance \\
\hline Age at BSIT (yrs), median, [range] & $64[28-76]$ & $64.5[58-71]$ & \\
\hline$<65$ & 8 & 2 & n.s \\
\hline$\geq 65$ & 7 & 2 & \\
\hline \multicolumn{4}{|l|}{ Gender } \\
\hline Female & 4 & 1 & n.s \\
\hline Male & 11 & 3 & \\
\hline \multicolumn{4}{|l|}{ Diagnostic accuracy } \\
\hline Definite AE & 13 & 1 & 0.037 \\
\hline Possible/probable AE & 2 & 3 & \\
\hline \multicolumn{4}{|l|}{ Antibody status } \\
\hline Seropositive & 8 & 1 & n.s \\
\hline Seronegative & 7 & 3 & \\
\hline Diagnostic delay (mo), median, [range] & $4[0.5-48]$ & $7[4.5-48]$ & \\
\hline$\leq 3$ & 7 & 0 & n.s \\
\hline$>3$ & 8 & 4 & \\
\hline Delay to BSIT (mo), mean, [range] & $42[6-96]$ & $39.75[11-96]$ & n.s \\
\hline Current smokers & 5 & 1 & n.s \\
\hline All-time smokers & 9 & 3 & n.s \\
\hline ENT comorbidities & 2 & 2 & n.s \\
\hline mRS at onset, median [range] & $3[1-5]$ & $1[1]$ & \\
\hline$<3$ & 5 & 4 & 0.033 \\
\hline$\geq 3$ & 10 & 0 & \\
\hline Cognitive impairment at onset & 12 & 1 & n.s \\
\hline Mood disorder at onset & 12 & 4 & n.s \\
\hline Behavioral disorders at onset & 6 & 0 & n.s \\
\hline Sleep disorders at onset & 8 & 1 & n.s \\
\hline \multicolumn{4}{|l|}{ Inflammatory $\mathrm{CSF}^{\S}$} \\
\hline Yes & 7 & 0 & n.s \\
\hline No & 6 & 3 & \\
\hline Not available & 2 & 1 & \\
\hline mRS at follow-up, median, [range] & $1[0-3]$ & 1 & n.s \\
\hline Autoimmune-associated epilepsy & 11 & 3 & n.s \\
\hline \multicolumn{4}{|l|}{ MoCA score at BSIT } \\
\hline$>26$ & 8 & 3 & n.s \\
\hline$\leq 26$ & 7 & 1 & \\
\hline Mood disorder at B-SIT & 9 & 1 & n.s \\
\hline \multicolumn{4}{|l|}{ MRI findings } \\
\hline Unilateral mT & 4 & 3 & n.s \\
\hline Bilateral mT & 10 & 1 & \\
\hline Not applicable & 1 & 0 & \\
\hline \multicolumn{4}{|l|}{ EEG abnormalities } \\
\hline Unilateral & 2 & 4 & 0.006 \\
\hline Bilateral & 11 & 0 & \\
\hline Not applicable & 2 & 0 & \\
\hline \multicolumn{4}{|l|}{ Response to IT } \\
\hline Yes/partial & 14 & 2 & n.s \\
\hline No/not applicable & 1 & 2 & \\
\hline
\end{tabular}

Bold indicates statistically significant results

$A E$ autoimmune encephalitis, $B$-SIT Brief Smell Identification Test, CSF cerebrospinal fluid, $E N T$ ear, nose and throat, IT immunotherapy, MoCA Montreal Cognitive Assessment, $m R S$ modified Rankin Scale, $m T$ mesial temporal

${ }^{\S}$ Inflammatory CSF changes were defined as elevated CSF protein level $>45 \mathrm{mg} / \mathrm{dl}$, lymphocytic pleocytosis $>5$ cells $/ \mathrm{mcL}$ 
To verify this hypothesis, we investigated the olfactory function of 19 patients previously diagnosed with $\mathrm{AE}$ through a validated forced-choice test, and found that $78.9 \%$ of them were either hyposmic or anosmic, a significantly higher percentage compared with sex- and age-matched HC $(15 / 19$ vs $5 / 19, p=0.0029)$. Our observation is consistent with a previous work by Geran et al. (5), documenting smell impairment in 24 out of $32(75 \%)$ AE cases. Therefore, our findings confirm that olfaction impairment is much more common in $\mathrm{AE}$ than in the general population, where its rate ranges from 3.8 to $25 \%$ [12-15]. The proportion found in our cohort (whose median age was 64 years) is remarkably high even when comparing it only to the elderly, whose smell loss is reportedly up to $50 \%$ due to olfaction physiological decline after the fourth decade of life [16]. Besides, in our population age did not appear as a determinant of olfactory deficit, based on statistical analysis.

In our study, smell impairment was more common in subjects with higher "acute" mRS scores, suggesting that these patients were severely affected at AE onset. Indeed, their diagnostic delay was shorter (although not significantly) compared with participants with normal olfaction, and they were also more often diagnosed with 'definite' AE. Therefore, it is probable that in severe cases, with a "full-blown" clinical presentation, the underlying intense immune-mediated processes significantly damage the central olfactory structures, resulting in a persistent smell deficit. In line with this hypothesis, we detected CSF inflammatory changes in a proportion of hyposmic patients (7/15) but not in normal smellers. Still, it is not possible to exclude that degenerative changes "triggered" by inflammatory/immune-mediated mechanisms might, in fact, cause olfaction to gradually decline over time in subjects with previous AE, as in other progressive disorders.

As to the Abs status, no difference was found between seropositive and seronegative cases. Unfortunately, none of our seronegative patients was tested for anti-AK5 Abs, which have been recently associated with smell loss [6]; still, based on phenotypical discrepancies (i.e. the prominence of epileptic manifestations in our population, which are rareand generally late-in anti-AK5 encephalitis), it appears unlikely that any of them actually harbored anti-AK5 Abs.

Finally, smell impairment was detected more frequently in cases where mesial temporal involvement was presumably bilateral, based on ictal/interictal EEG findings (which could reveal a wider brain participation than detected through structural neuroimaging studies alone) [17]. This observation leads to further reflection upon the controversial topic of olfactory function lateralization. Indeed, studies performed in subjects with mesial temporal lobe epilepsy (MTLE) documented smell impairment in patients with unilateral as well as bilateral TLE, irrespective of the side of seizure focus, suggesting that both hemispheres are important in olfactory processing [18], although recent evidence points to a "disproportionate role" of the right one [19]. Considering both our findings and literature data, we could hypothesize that, despite the predominance of ipsilateral connections among primary central olfactory structures, alterations in smell processing are more likely to become clinically "evident" when fronto-temporal areas are bilaterally affected. However, delivering the olfactory stimuli birhinally, as we did, might also have influenced the patients' performance.

This study has several limitations, first the small sample size. Besides, B-SIT allows to evaluate odor identification (which exemplifies semantic odor memory), but alterations in odor perception and discrimination cannot be properly assessed and ruled out, especially in the minority of subjects spontaneously complaining of smell alterations. This also prevents us from attempting any specific anatomoclinical correlation: in particular, we cannot exclude that an immune-mediated damage to the peripheral olfactory structures contributes to our patients' impairment, although its central origin appears more likely. Finally, the cognitive deficits and mood changes that were found in a remarkable proportion of the study participants might have influenced their performance at B-SIT.

In conclusion, our work confirms that olfactory dysfunction is common-although usually undiagnosed-in AE patients, and suggests that smell tests should be routinely performed when immune-mediated encephalitis is suspected. Further studies are warranted to elucidate the exact extent and evolution of olfaction impairment, which might represent an additional-and interesting — piece of the complex puzzle of AE.

Supplementary Information The online version contains supplementary material available at https://doi.org/10.1007/s00415-022-10959-6.

Acknowledgements The authors would like to thank Dr Stefano Bucci for performing anterior rhinoscopy. G.R. was supported by BE-FORERC program (Sapienza University) and Italian Ministry of Health "Ricerca corrente".

Funding This research did not receive any specific grant from funding agencies in the public, commercial, or not-for-profit sectors.

Availability of data Data are available from the corresponding author, upon reasonable request.

\section{Declarations}

Conflicts of interest No competing interests to declare.

Ethical approval The study was undertaken with the understanding and written consent of each subject, and conforms with World Medical Association Declaration of Helsinki. 


\section{References}

1. Hoover KC (2010) Smell with inspiration: the evolutionary significance of olfaction. Am J Phys Anthropol 143(Suppl 51):63-74. https://doi.org/10.1002/ajpa.21441

2. Moein ST, Hashemian SM, Mansourafshar B, Khorram-Tousi A, Tabarsi P, Doty RL (2020) Smell dysfunction: a biomarker for COVID-19. Int Forum Allergy Rhinol 10(8):944-950. https://doi. org/10.1002/alr.22587

3. Doty RL (2017) Olfactory dysfunction in neurodegenerative diseases: is there a common pathological substrate? Lancet Neurol 16(6):478-488. https://doi.org/10.1016/S1474-4422(17)30123-0

4. Shin T, Kim J, Ahn M, Moon C (2019) Olfactory dysfunction in CNS neuroimmunological disorders: a review. Mol Neurobiol 56(5):3714-3721. https://doi.org/10.1007/s12035-018-1341-0

5. Geran R, Uecker FC, Prüss H, Haeusler KG, Paul F, Ruprecht K et al (2019) Olfactory and gustatory dysfunction in patients with autoimmune encephalitis. Front Neurol 14(10):480. https://doi. org/10.3389/fneur.2019.00480

6. Muñiz-Castrillo S, Hedou JJ, Ambati A, Jones D, Vogrig A, Pinto AL et al (2021) Distinctive clinical presentation and pathogenic specificities of anti-AK5 encephalitis. Brain. https://doi.org/10. 1093/brain/awab153 (Online ahead of print)

7. Graus F, Titulaer MJ, Balu R, Benseler S, Bien CG, Cellucci $\mathrm{T}$ et al (2016) A clinical approach to diagnosis of autoimmune encephalitis. Lancet Neurol 15(4):391-404

8. Dintica CS, Marseglia A, Rizzuto D, Wang R, Seubert J, Arfanakis K, Bennett DA, Xu W (2019) Impaired olfaction is associated with cognitive decline and neurodegeneration in the brain. Neurology 92(7):e700-e709. https://doi.org/10.1212/WNL.0000000000 006919

9. Nasreddine ZS, Phillips NA, Bédirian V, Charbonneau S, Whitehead V, Collin I et al (2005) The Montreal Cognitive Assessment, MoCA: a brief screening tool for mild cognitive impairment. J Am Geriatr Soc 53(4):695-699

10. Avorio F, Morano A, Fanella M, Fattouch J, Albini M, Basili LM et al (2019) Olfactory stimulus-induced temporal lobe seizures in limbic encephalitis. Seizure 69:204-206. https://doi.org/10.1016/j. seizure.2019.05.005

11. Schub FR, Cain WS (1991) Memory for odors. In: Doty RL, Breiphol W (eds) The human sense of smell. Springer-Verlag Berlin Heid, New York, p 395

12. Murphy C, Schubert CR, Cruickshanks KJ, Klein BE, Klein R, Nondahl DM (2002) Prevalence of olfactory impairment in older adults. JAMA 288(18):2307-2312. https://doi.org/10.1001/jama. 288.18.2307

13. Vennemann MM, Hummel T, Berger K (2008) The association between smoking and smell and taste impairment in the general population. J Neurol 255(8):1121-1126. https://doi.org/10.1007/ s00415-008-0807-9

14. Schubert CR, Cruickshanks KJ, Fischer ME, Huang GH, Klein BE, Klein R et al (2012) Olfactory impairment in an adult population: the Beaver Dam Offspring Study. Chem Senses 37(4):325334. https://doi.org/10.1093/chemse/bjr102

15. Yang J, Pinto JM (2016) The epidemiology of olfactory disorders. Curr Otorhinolaryngol Rep 4(2):130-141

16. Doty RL, Shaman P, Applebaum SL, Giberson R, Siksorski L, Rosenberg L (1984) Smell identification ability: changes with age. Science 226(4681):1441-1443. https://doi.org/10.1126/science. 6505700

17. Morano A, Fanella M, Cerulli Irelli E, Barone FA, Fisco G, Orlando B et al (2020) Seizures in autoimmune encephalitis: findings from an EEG pooled analysis. Seizure 83:160-168. https:// doi.org/10.1016/j.seizure.2020.10.019

18. Desai M, Agadi JB, Karthik N, Praveenkumar S, Netto AB (2015) Olfactory abnormalities in temporal lobe epilepsy. J Clin Neurosci 22(10):1614-1618. https://doi.org/10.1016/j.jocn.2015.03.035

19. Doty RL, Tourbier I, Neff JK, Silas J, Turetsky B, Moberg P et al (2018) Influences of temporal lobe epilepsy and temporal lobe resection on olfaction. J Neurol 265(7):1654-1665. https://doi. org/10.1007/s00415-018-8891-y 